\title{
A Natural Interaction Interface for UAVs Using Intuitive Gesture Recognition
}

\author{
Meghan Chandarana ${ }^{1}$ \\ Anna Trujillo² \\ Kenji Shimada ${ }^{1}$ \\ Danette Allen ${ }^{2}$
}

${ }^{1}$ Carnegie Mellon University, Pittsburgh, PA

${ }^{2}$ NASA Langley Research Center, Hampton, VA

AIAA Aviation 2016

June 16, 2016 


\section{Outline}

1. Objectives

2. Motivation

3. Gesture Sensor

- Leap Motion

4. Ground Control System

- Volume Definition

- Gesture Module

- Trajectory Generation

- Validation

5. Demo Video

6. Future Work

7. Conclusion 


\section{Objectives}

Develop a gesture-based natural language interface which nonexpert users can quickly and easily use to build a desired flight path for an autonomous UAV by defining trajectory segments.

System Considerations:

1. Intuitive natural interaction

- Similar to speaking with another person

2. Simple interface flow

3. Ample feedback for decision making

4. User concerned only with high-level mission definition

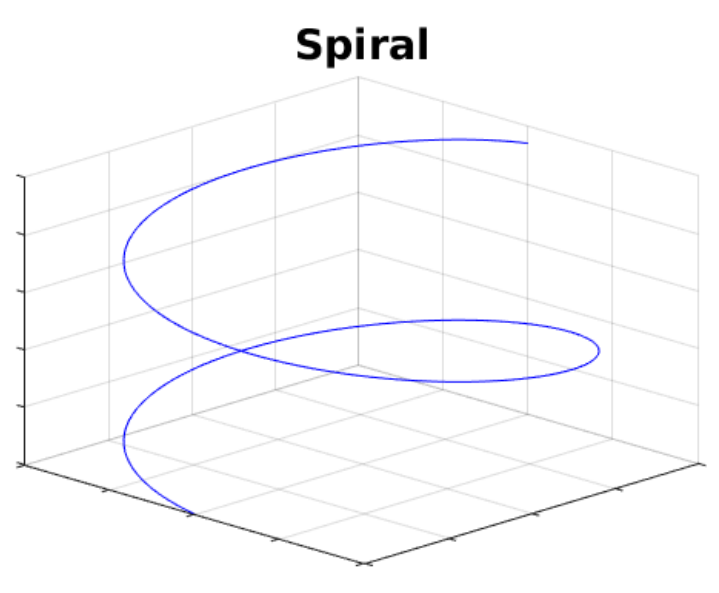




\section{Atmospheric Science Mission}



Mission Parameters

- Fly multiple vehicles

- Correlative data acquisition

- Intuitive interface

- DO NOT need low-level understanding of architecture 


\section{Leap Motion Controller}

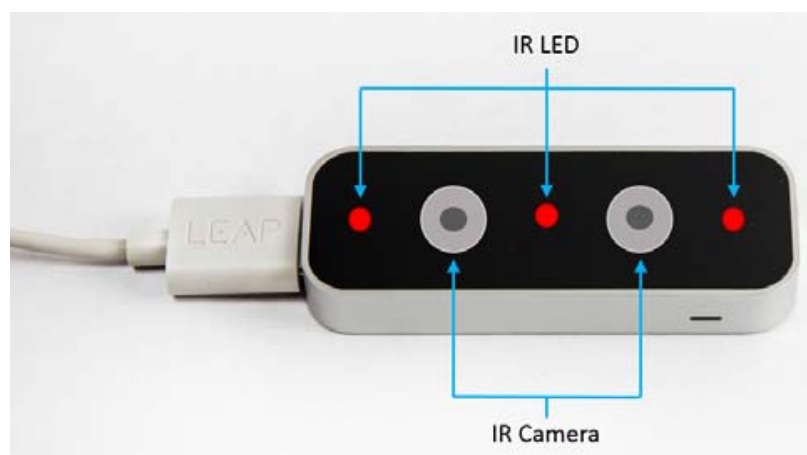

- Infrared cameras

- 200 frames per second

- $150^{\circ}$ field of view

- $8 \mathrm{ft}^{3}$ of interactive space

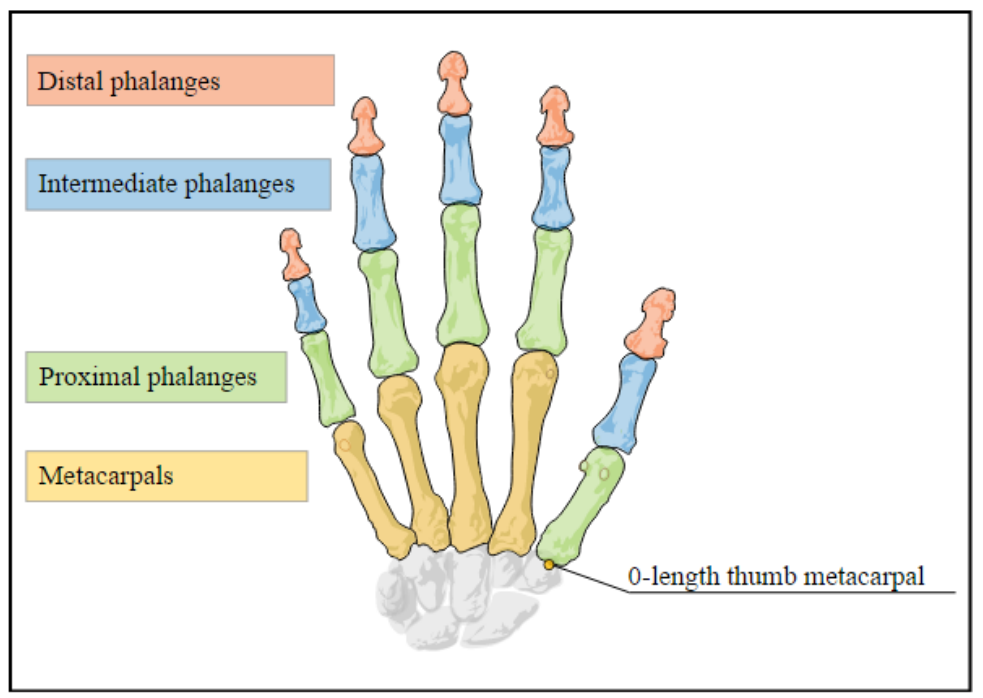




\section{Ground Control System}

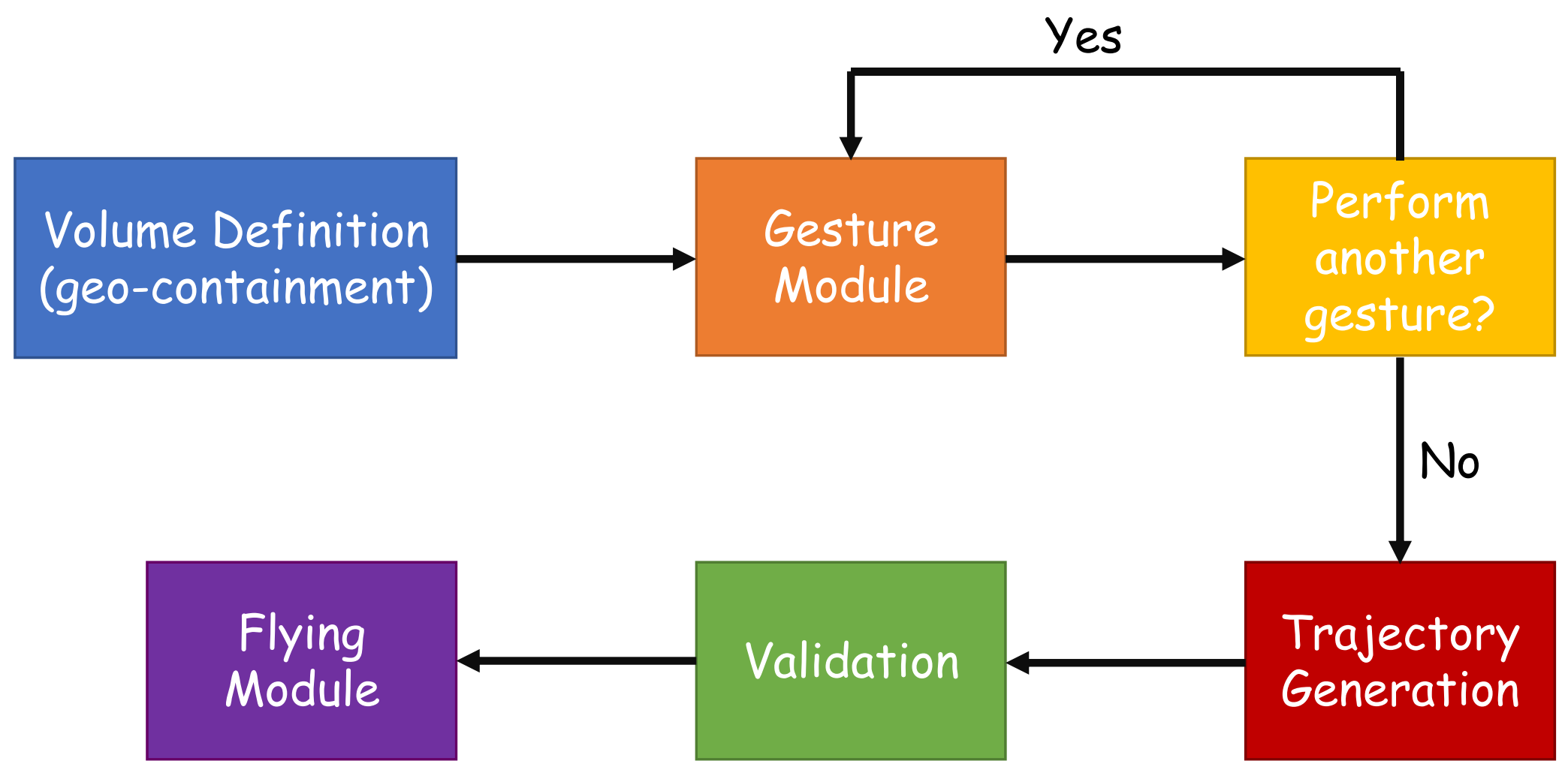




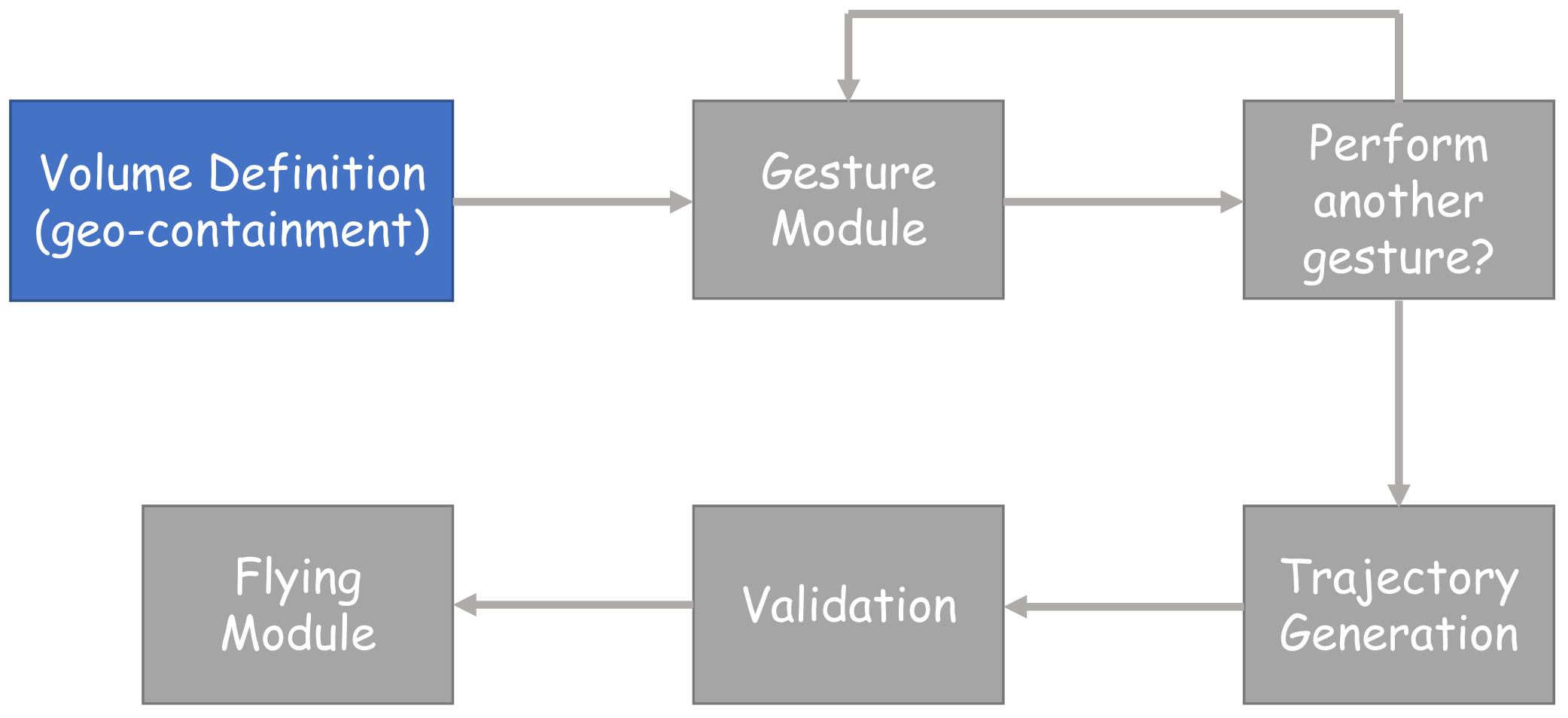




\section{Volume Definition}
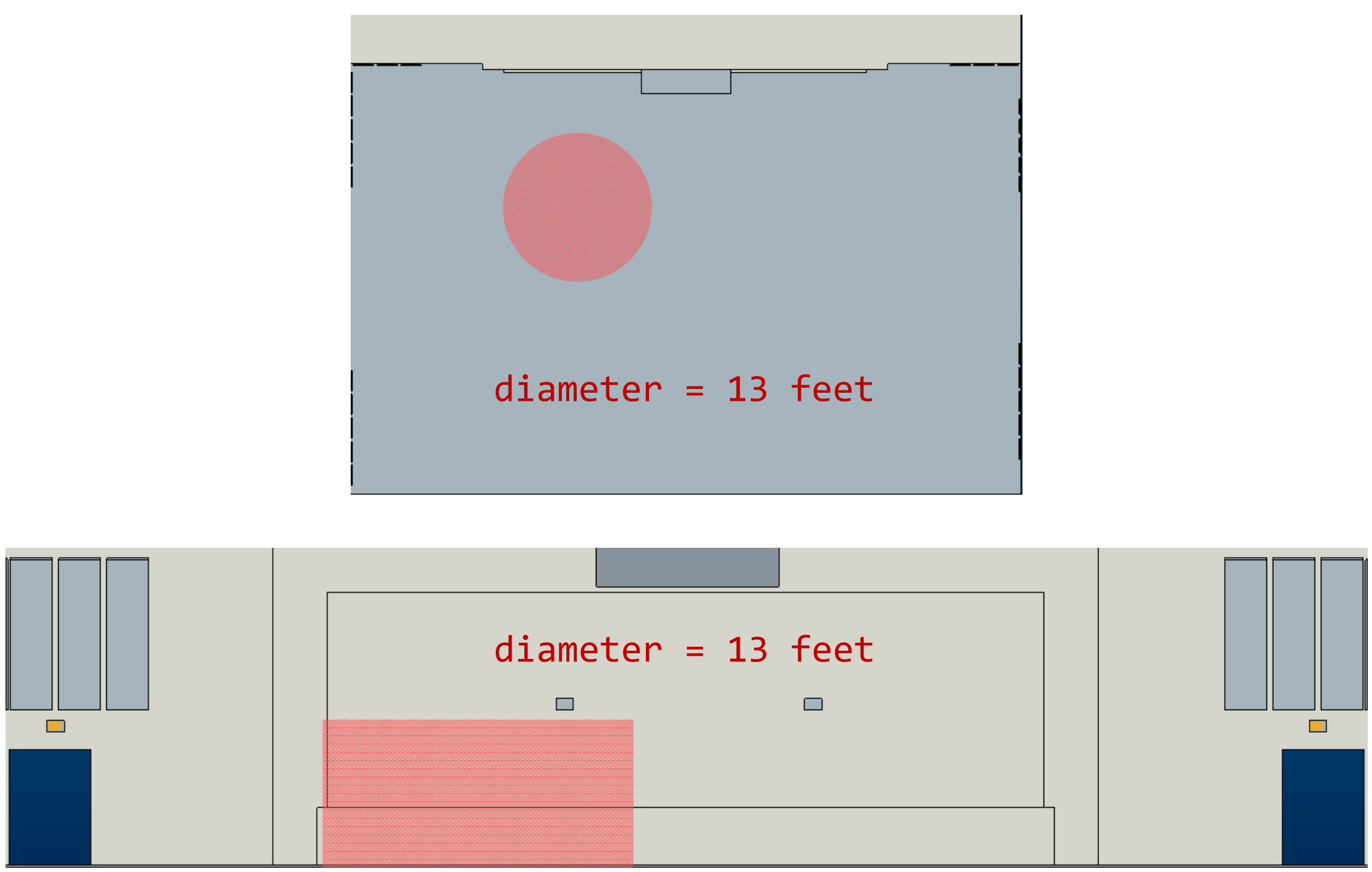


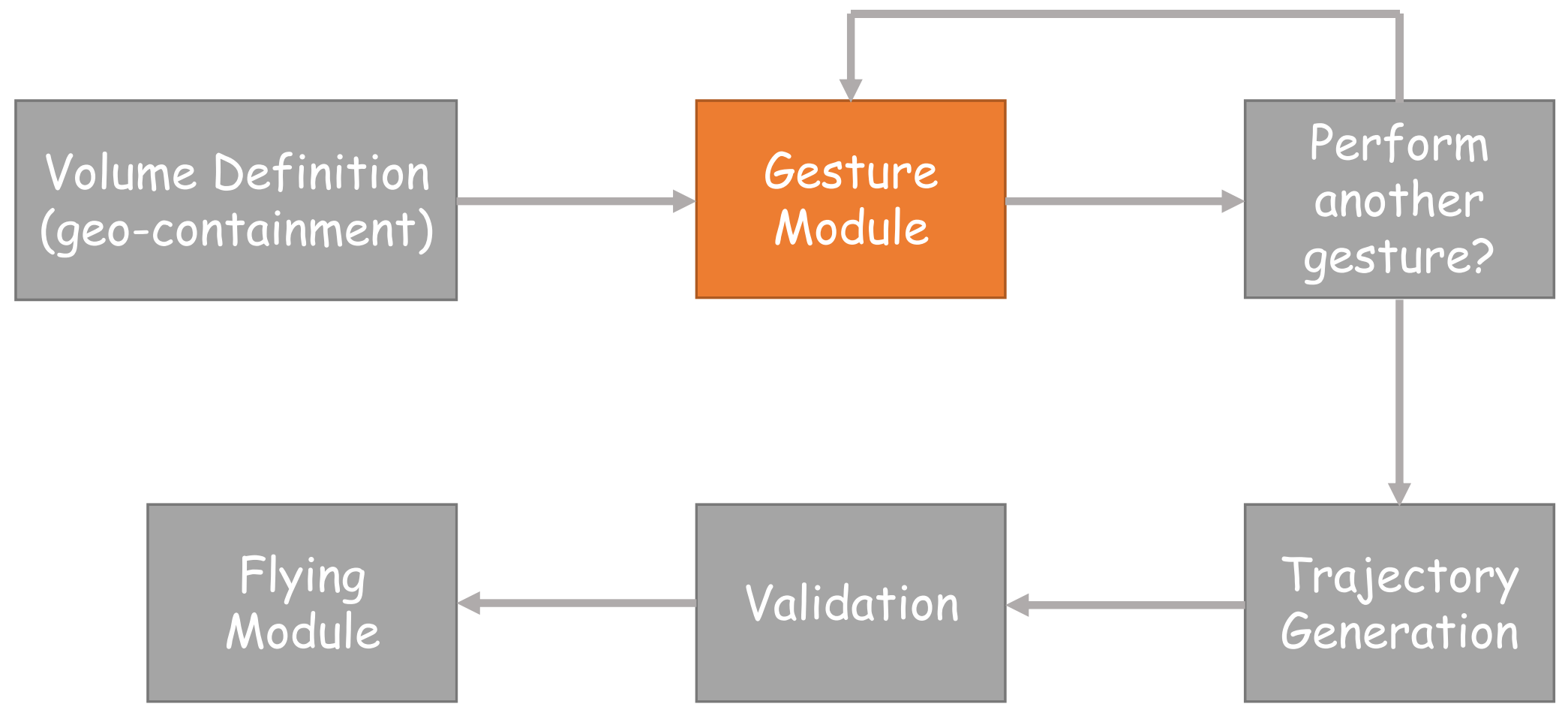




\section{Gesture Library}
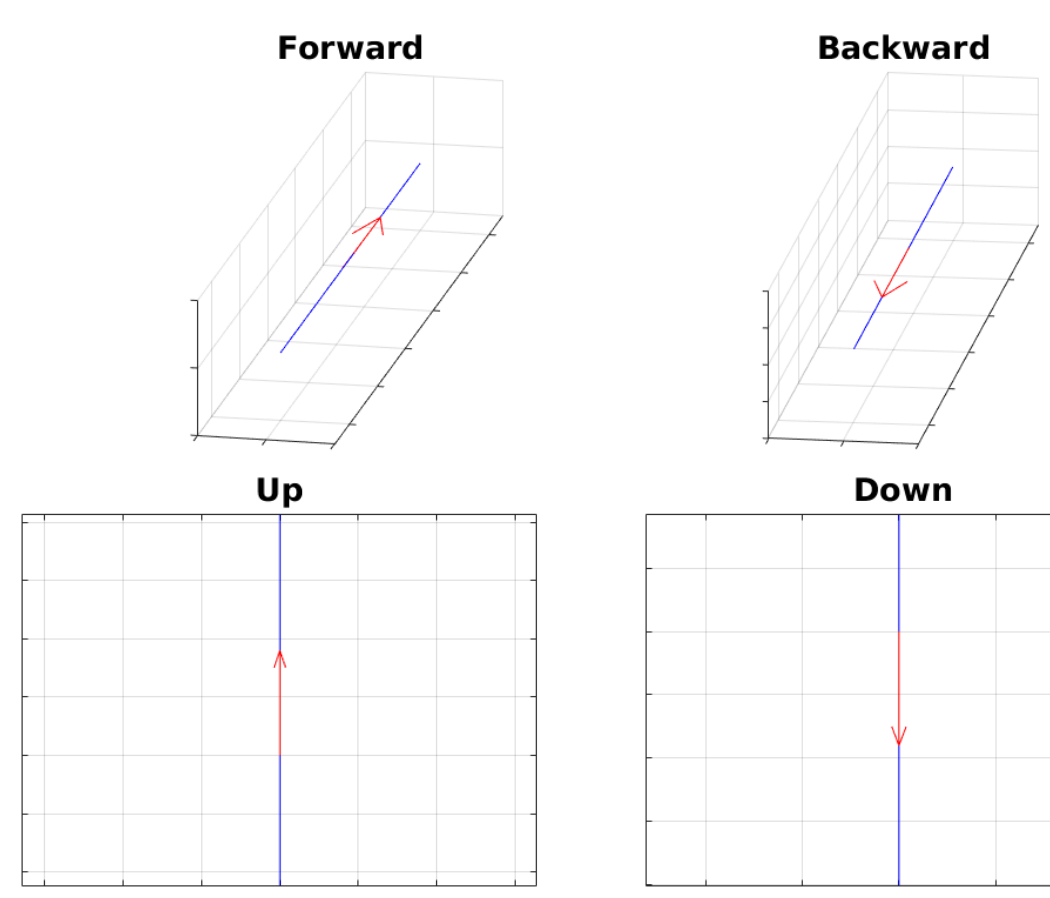

Backward-Left

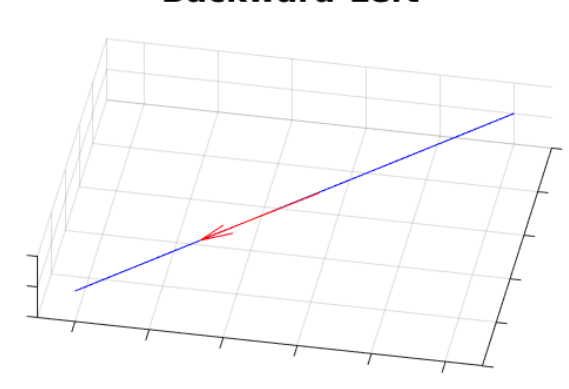

\section{Doum}

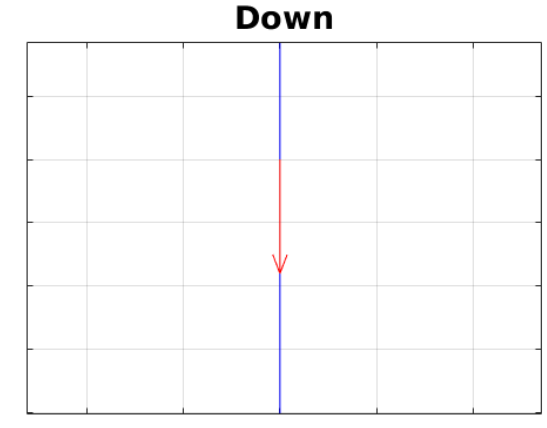

Backward-Right

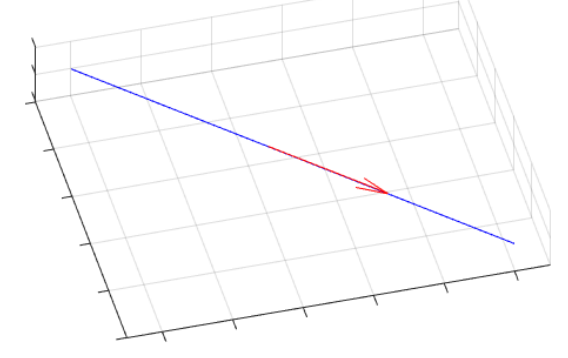

Right

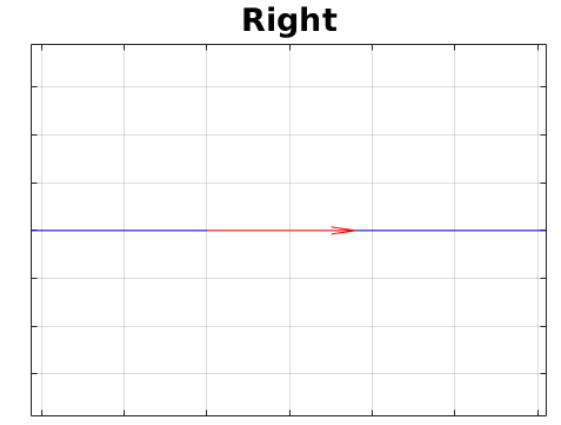

Forward-Left

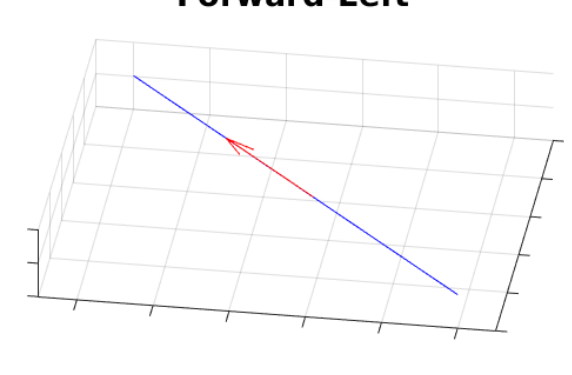

Circle

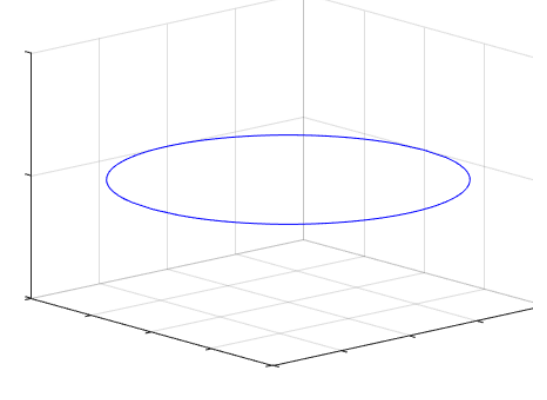

Left

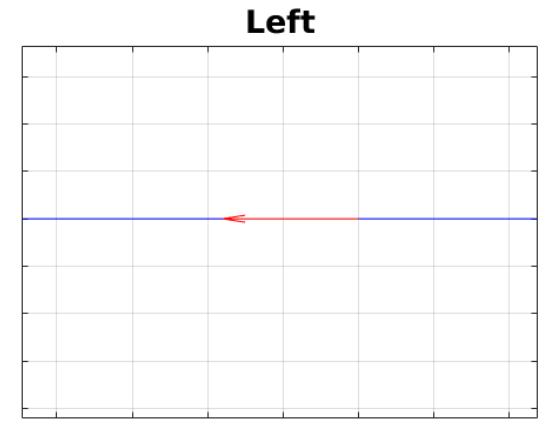

Forward-Right

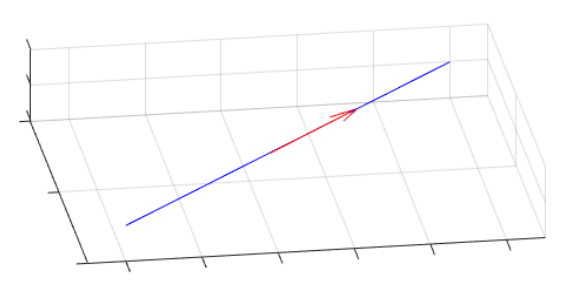

Spiral






\section{D Gesture Characterization}

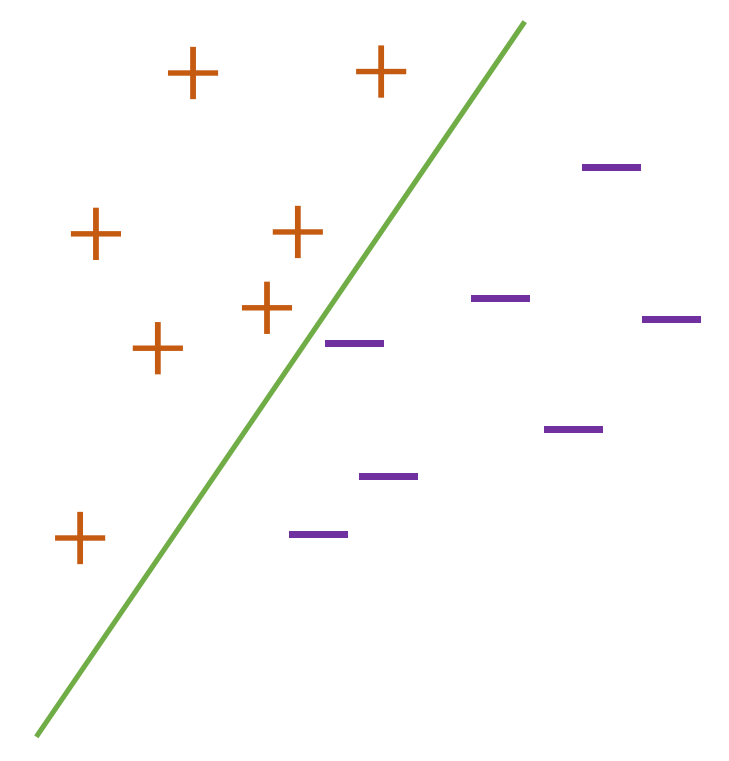

- Linear Support Vector Machine (SVM) Classifier

- 11 Subjects

- 10 samples per gesture

- Features used:

- Hand movement direction

- Eigenvalues 


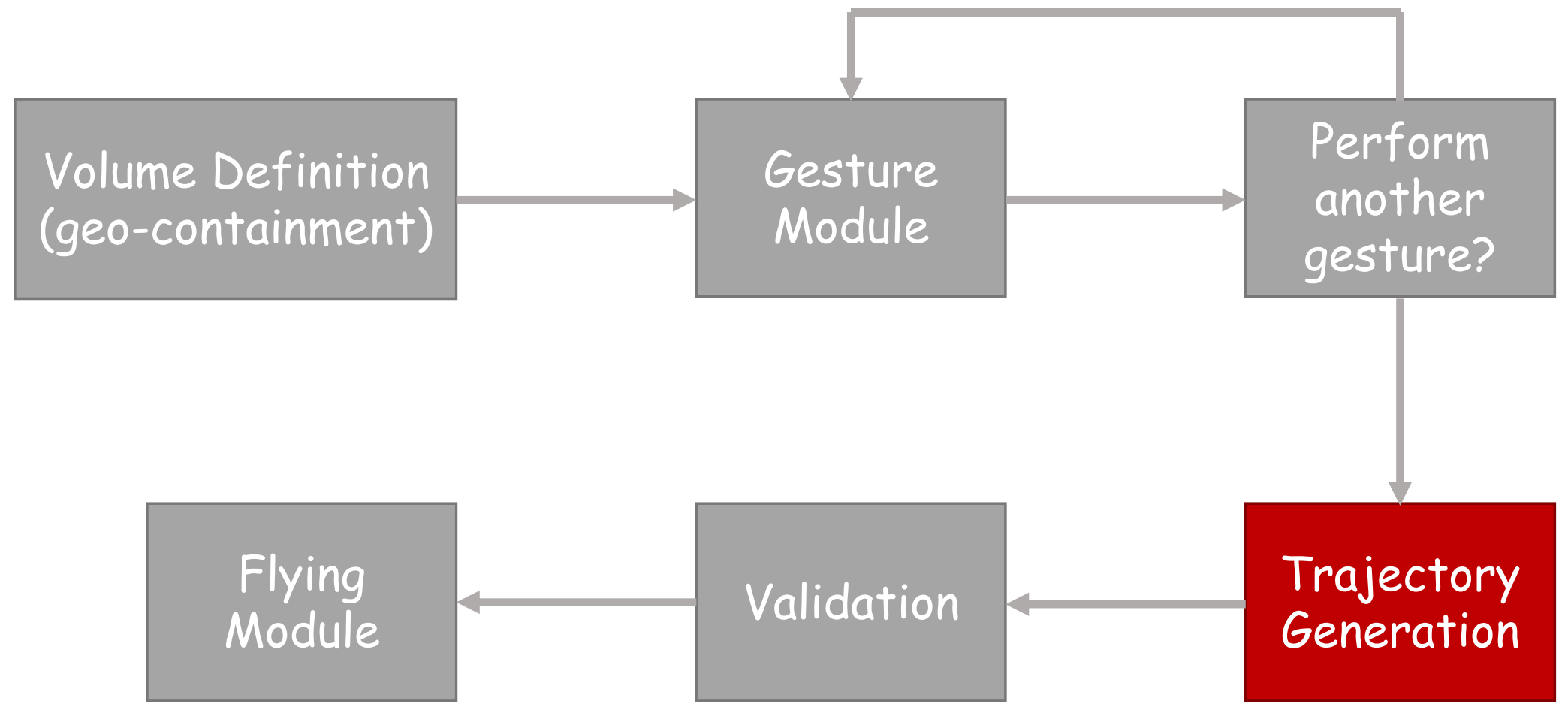




\section{Trajectory Generation}

- Translate gesture input segments into sets of Bézier splines

- Combine spline sets into a complete trajectory

- Smoothen spline transitions

- Transmit generated trajectory to vehicle controller

$$
B(t)=\sum_{i=0}^{n}\left(\begin{array}{l}
n \\
i
\end{array}\right)(1-t)^{n-i} t^{i} P_{i}
$$

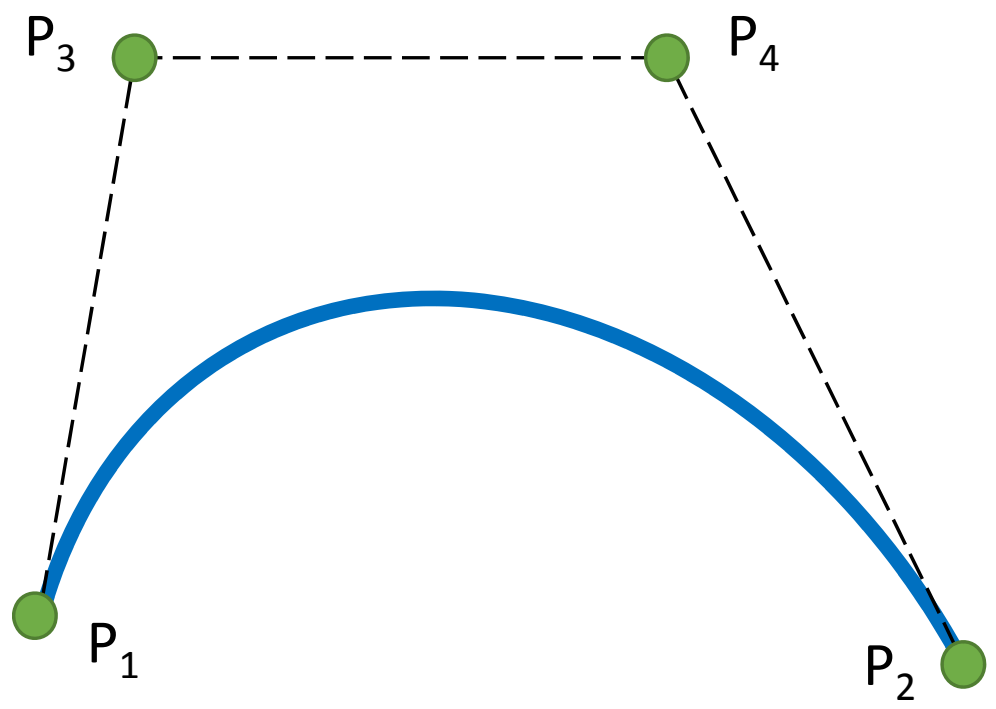




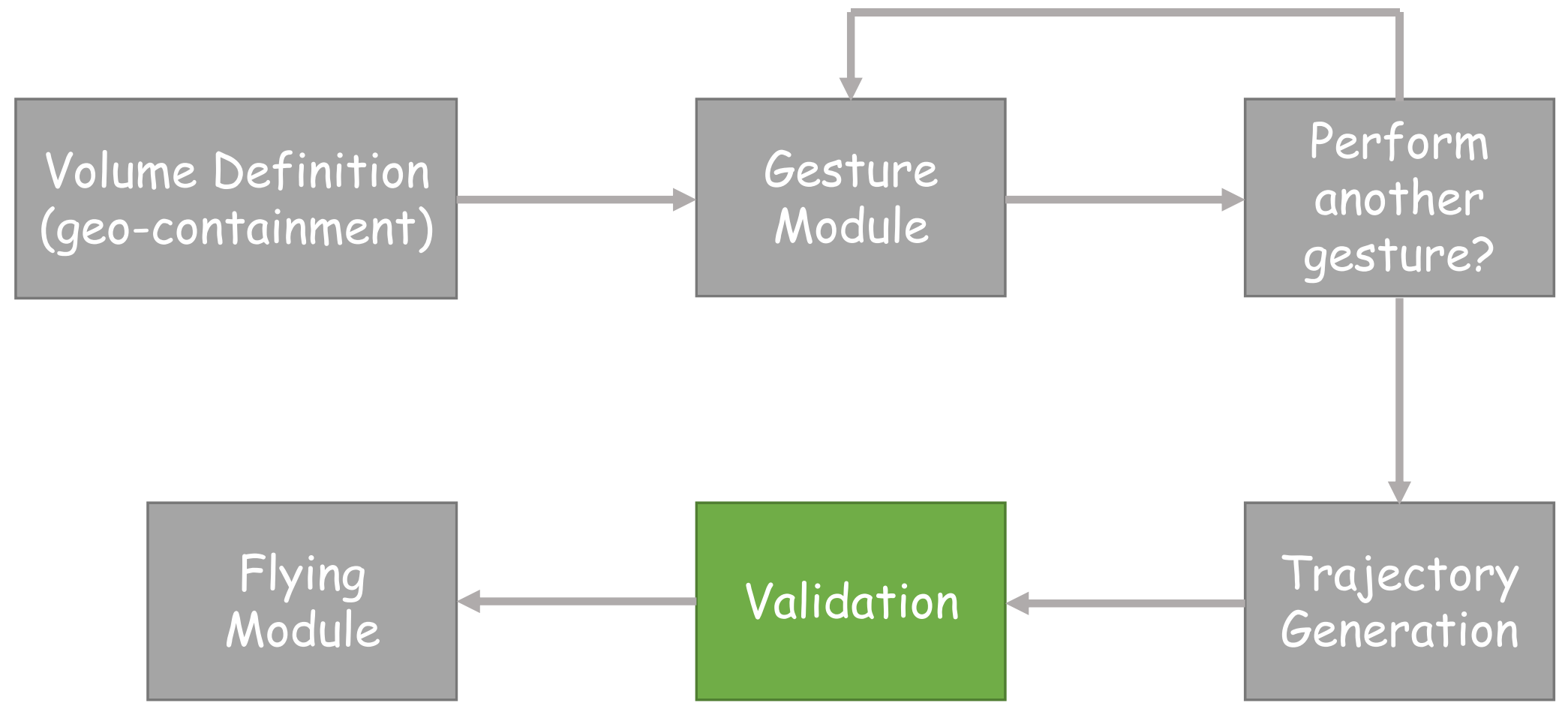




\section{Validation}


Sample Generated Trajectory for Validation 


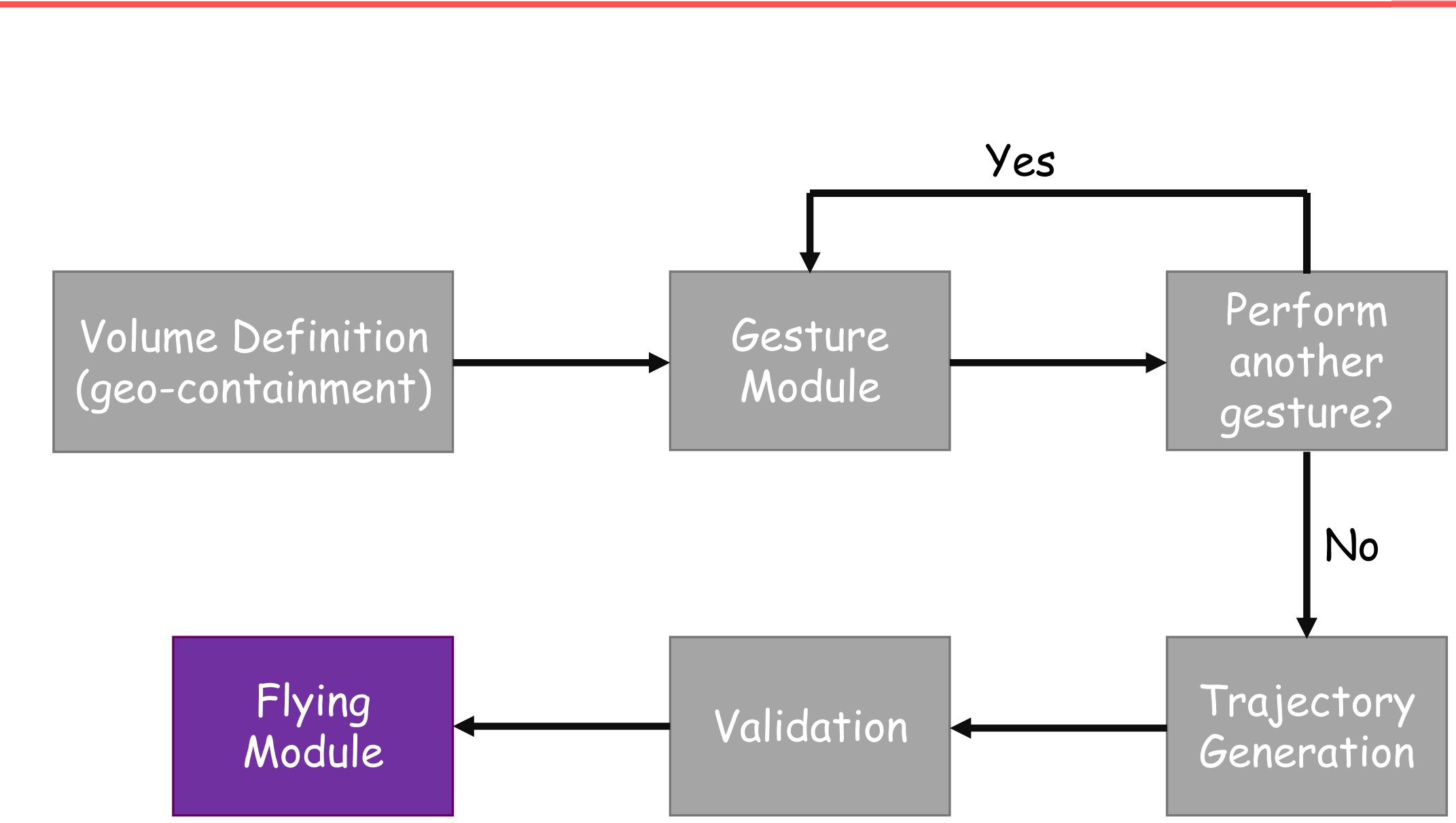




\section{Flight Module}

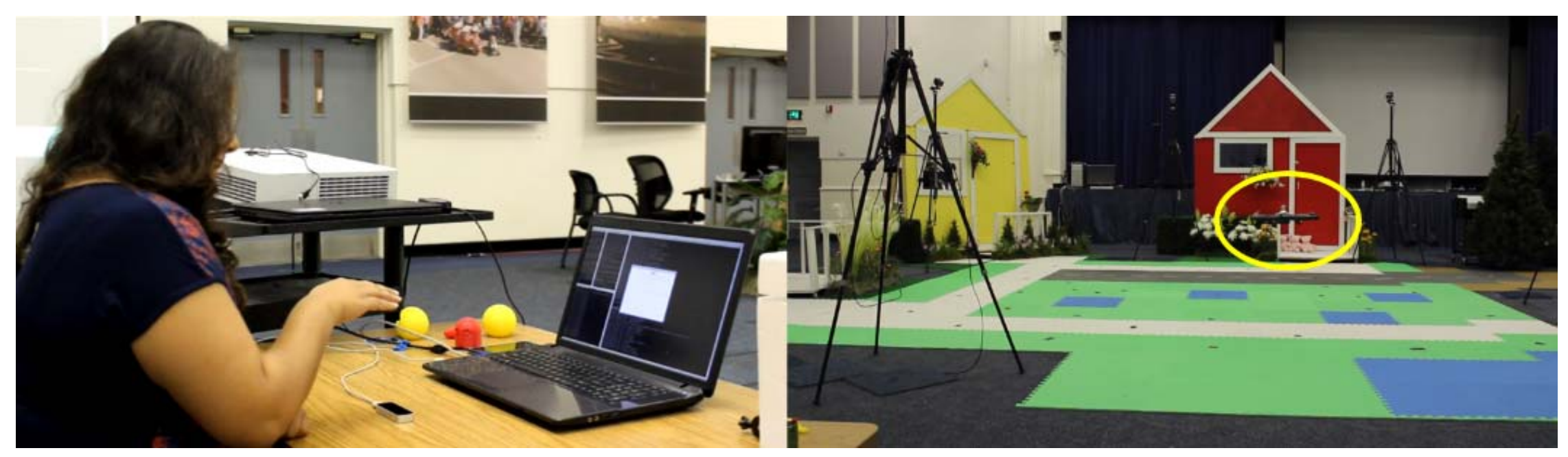

- Operator initiated flight

- Take off - "Up" gesture

- Land - "Down" gesture

- Commands sent directly to UAV controller

- Once in the air, the UAV automatically traverses defined flight path

- Hover and waits for land command at end of flight path 


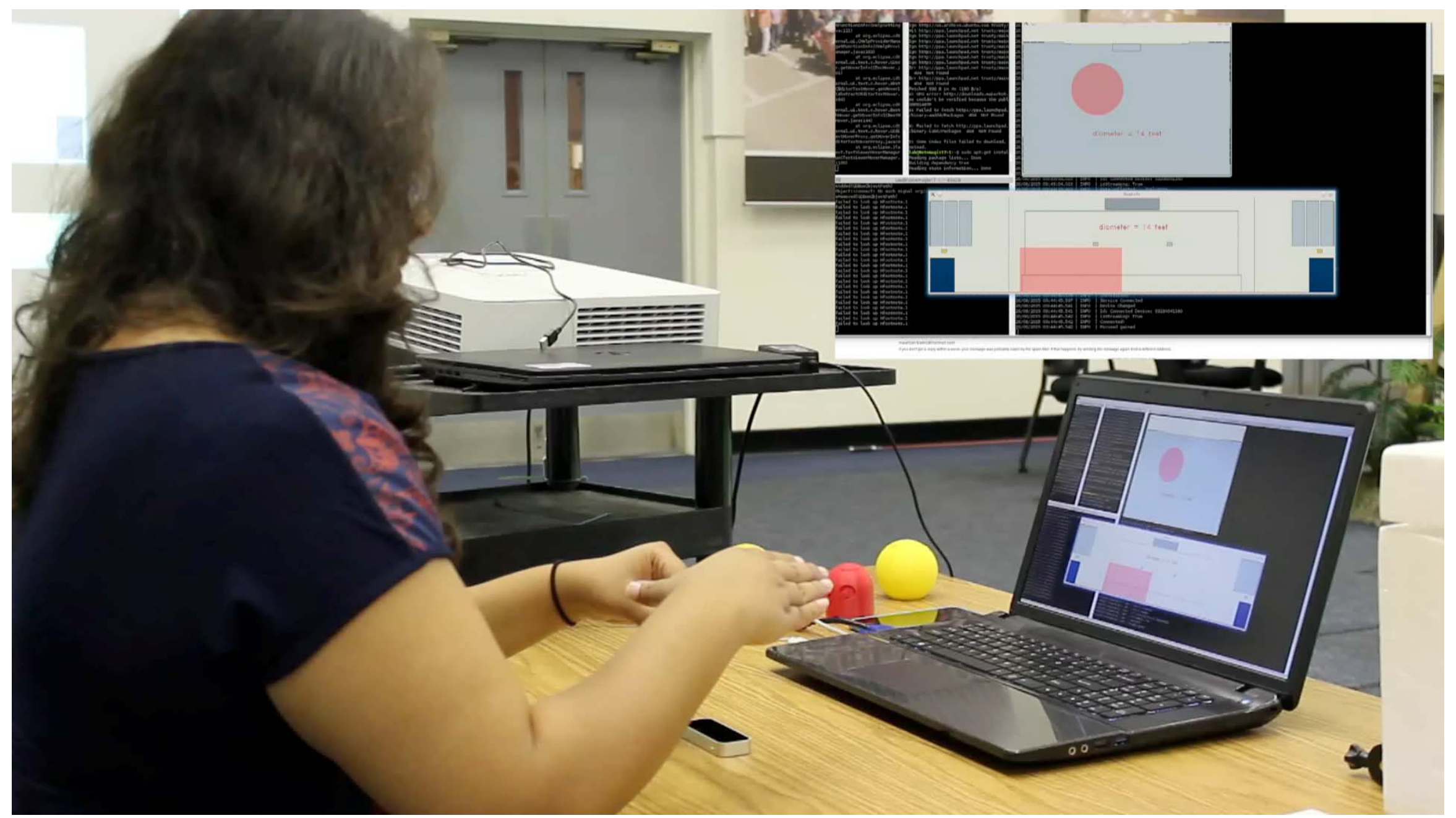




\section{Sample Flight Path}

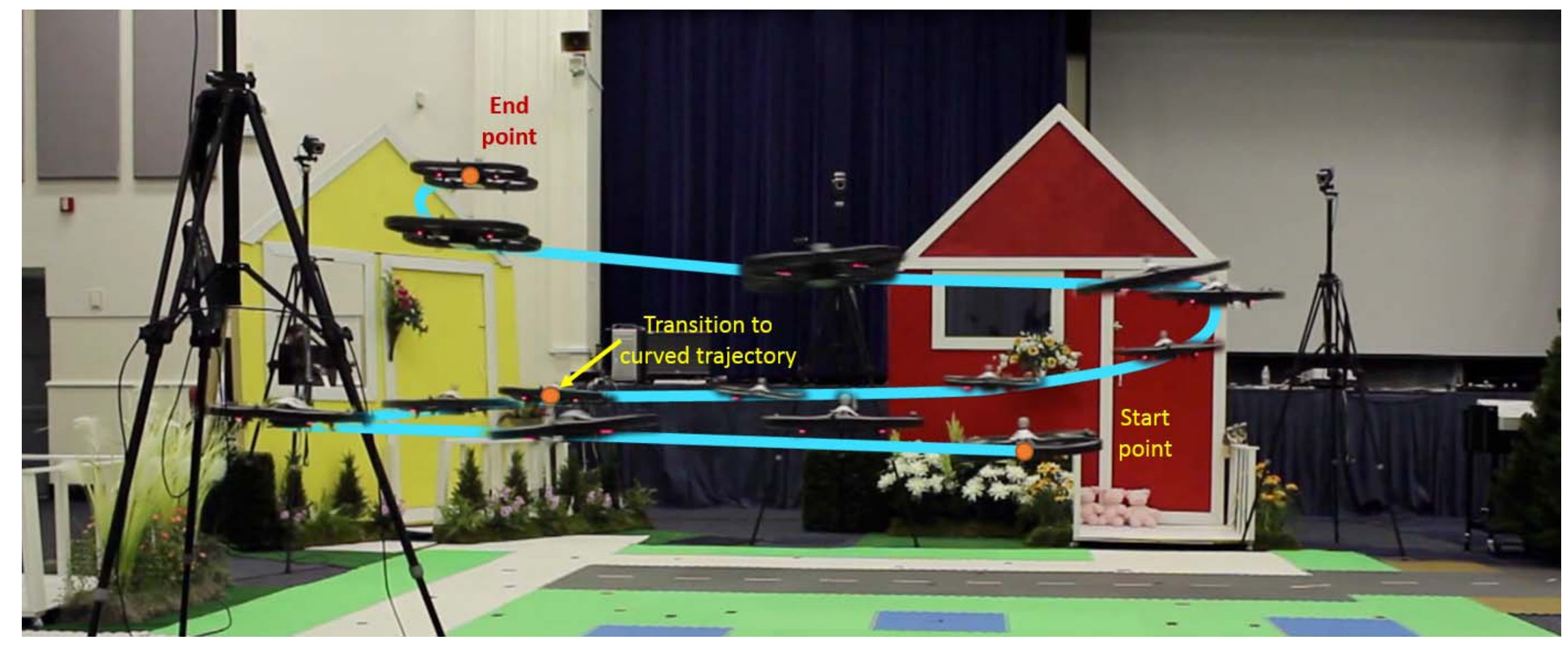




\section{Potential Future System}

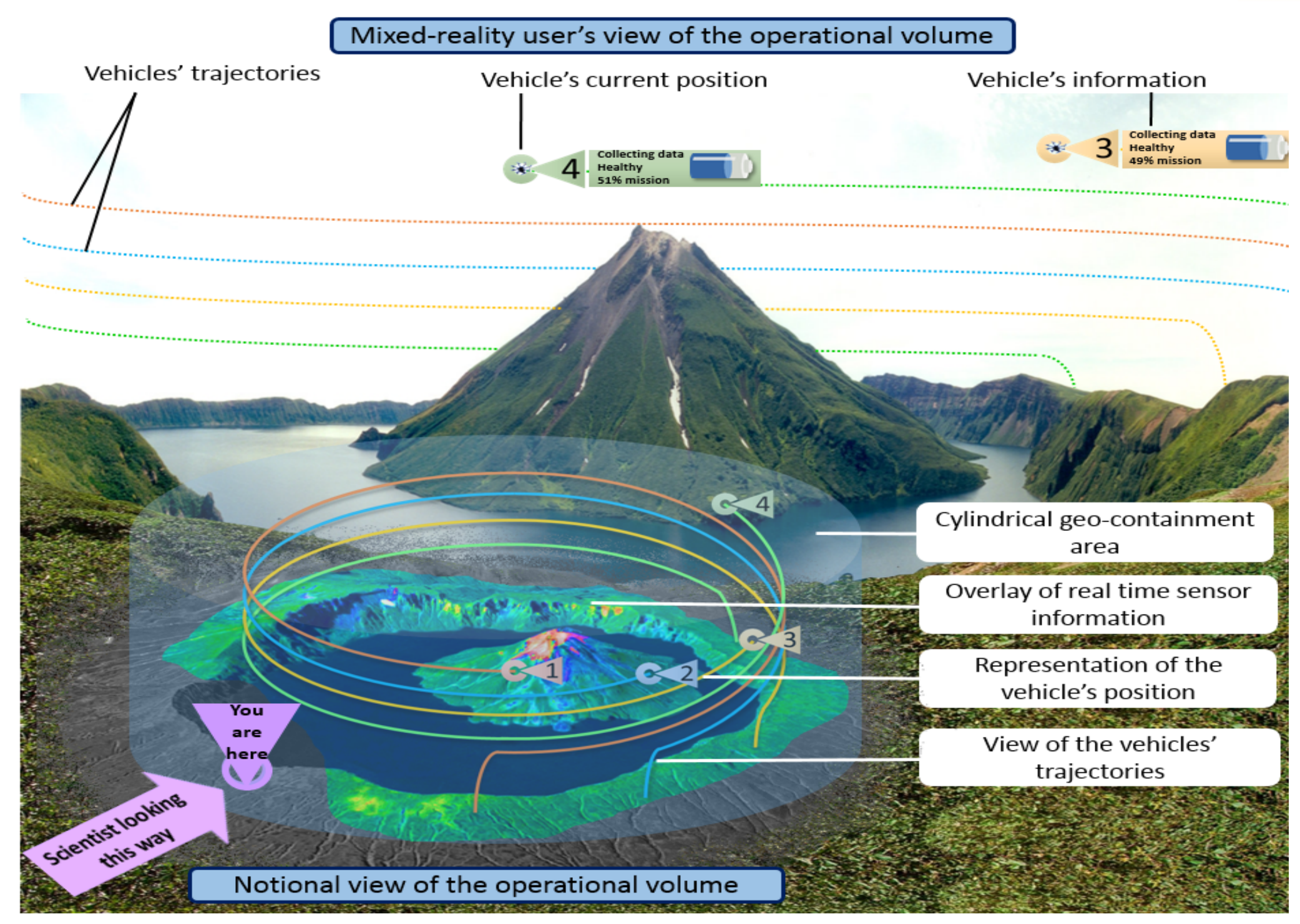




\section{Future Work}

1. The interface can be extended to include the definition of additional geometric constraints if necessary (e.g., clockwise vs counterclockwise direction on a circle).

2. The gesture library can be extended (e.g., spiral forward).

3.Gesture segmentation: A user may wish to define a complete square shape instead of teaching each segment one-by-one.

4. Extending the system to include real-time mission supervision and trajectory modification.

5.Perform user studies to fully validate the methodology. 


\section{Conclusion}

- Fully functioning end-to-end Ground Control System for defining trajectories for unmanned vehicles

- Intuitive, natural language interface

- Gesture Library

- Robust gesture characterization

- Feedback mechanisms at every step

\section{User does NOT need to understand low-level system architecture}




\title{
Acknowledgements
}

\author{
Dr. Danette Allen \\ Anna Trujillo \\ Javier Puig-Navarro \\ Bilal Mehdi \\ Gil Montague \\ Ben Kelley \\ Dr. Loc Tran \\ AI Team
}




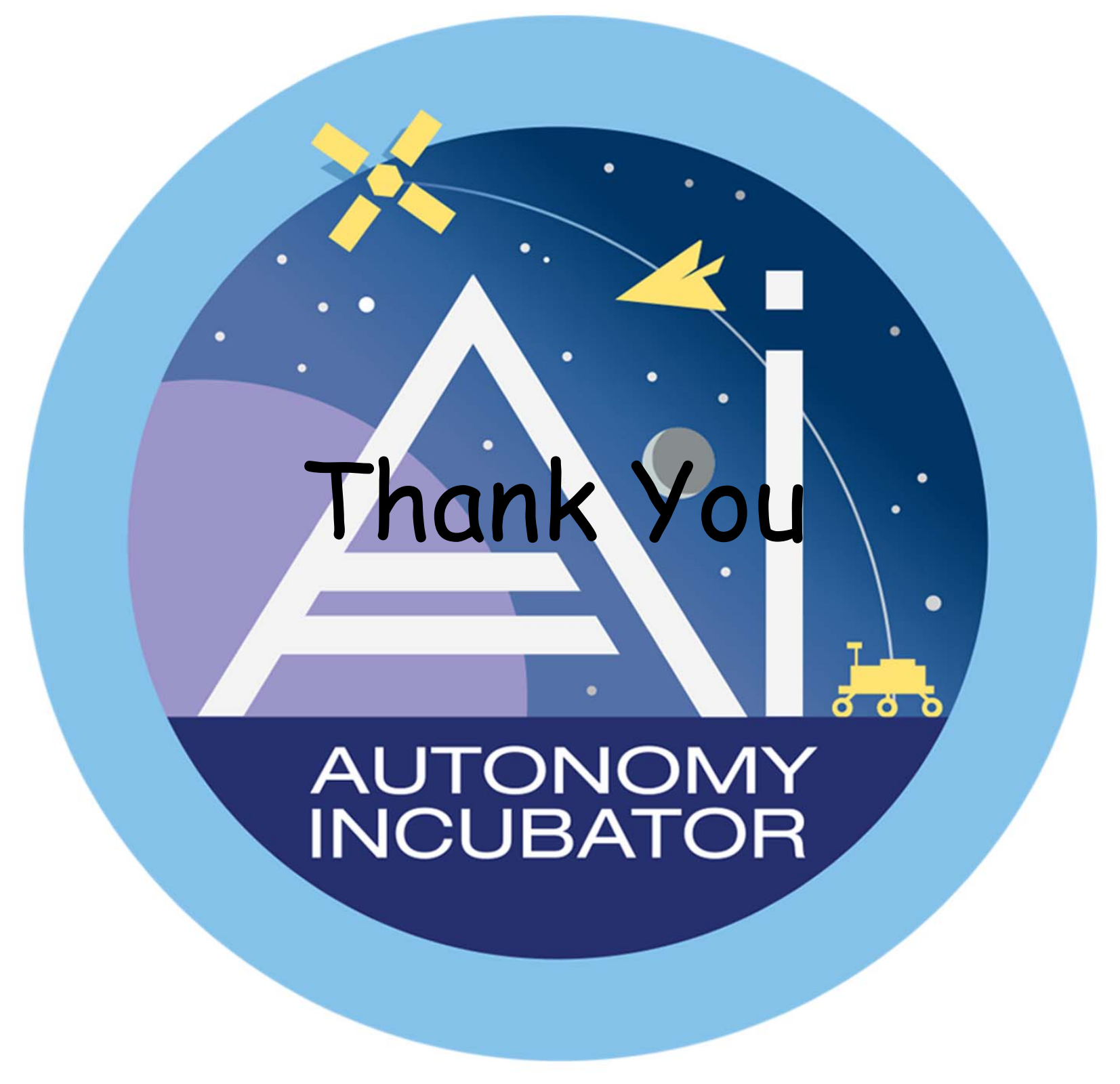

